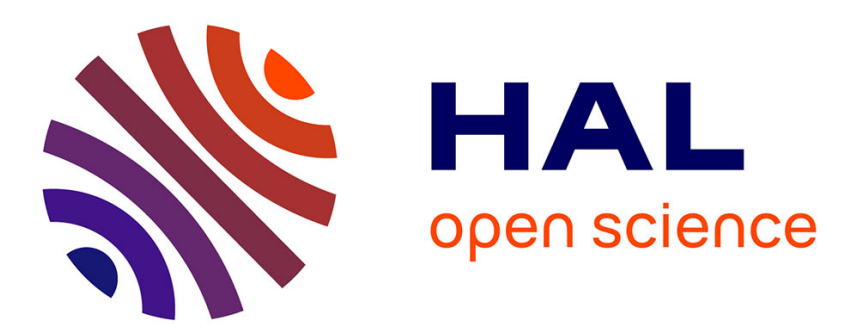

\title{
A Bayesian network for diagnosis of networked mobile robots
}

\author{
Insaf Sassi, Alexia Gouin, Jean-Marc Thiriet
}

\section{To cite this version:}

Insaf Sassi, Alexia Gouin, Jean-Marc Thiriet. A Bayesian network for diagnosis of networked mobile robots. ESREL 2016 - 26th European Safety and Reliability Conference, Sep 2016, Glasgow, United Kingdom. hal-01375924

\section{HAL Id: hal-01375924 \\ https://hal.science/hal-01375924}

Submitted on 7 Oct 2016

HAL is a multi-disciplinary open access archive for the deposit and dissemination of scientific research documents, whether they are published or not. The documents may come from teaching and research institutions in France or abroad, or from public or private research centers.
L'archive ouverte pluridisciplinaire HAL, est destinée au dépôt et à la diffusion de documents scientifiques de niveau recherche, publiés ou non, émanant des établissements d'enseignement et de recherche français ou étrangers, des laboratoires publics ou privés. 


\title{
A Bayesian network for diagnosis of networked mobile robots
}

\author{
I. Sassi, A. Gouin \& JM. Thiriet \\ Univ. Grenoble Alpes, GIPSA-lab, F-38000 Grenoble, France \\ CNRS, GIPSA-lab, F-38000 Grenoble, France
}

\begin{abstract}
The network of communicating mobile vehicles is a subclass of wireless networked control systems (WNCS) characterized by wireless communications and mobile nodes. The integration of the wireless network into control loop, given the stochastic aspects of wireless communication and mobility of its communicating entities, can lead to problems that affect system performances. In other words, the system quality of control QoC depends on the wireless network quality of service QoS state. A diagnosis method is essential to monitor, diagnose and maintain the system in an operational state. The present paper proposes a modular multi-layer Bayesian network model for diagnosis taking into account the network failures. Results regarding the system performance are presented to illustrate the relevance of the developed Bayesian Network BN to decisions making in order to lead the system to its final goal.
\end{abstract}

\section{INTRODUCTION}

Networked systems have emerged in many fields and in a large number of distributed applications. They are distributed systems where actuators, sensors and controllers are connected through a communication network which is crucial to ensure proper operation. Reducing the installation costs and the simplicity of maintenance and diagnosis of networked control systems prove the advantage of using these systems ( $\mathrm{Si}$ mon et al. 2013). Networked mobile robots, as wireless networked control systems WNCS, have found applications in transport field, domestic help, military, space, etc. They perform human tasks and they also take over human missions in hostile places, for instance robots that entered to the melted Fukushima reactor (Nagatani et al. 2013). The networked robots receive orders from a controller in order to accomplish their tasks and then they send back information about their state and other observations. For example, for the case of robot Philae, sent to the Tchouri comet to discover the evolution of solar system, researchers need to have a sufficient duration of communications, if it is possible, to receive a feedback from the robot and send him the orders to be executed. However, it is difficult to ensure that the data is transmitted correctly and completely because of the stochastic behavior of wireless network and the mobility of the communicating devices. The integration of wireless network into the control loop introduce problems that affect the system performance and its stability. In fact, the network state in term of quality of service QoS af- fects the system quality of control QoC. This influence is due to delay (Simon et al. 2013), (Ghostine et al. 2011), (Tipsuwan and Chow 2003), packets loss (Mechraoui et al. 2009), (Lian et al. 2002), (Berbra et al. 2008), jitter (Moyne and Tilbury 2007), throughput (Zhang et al. 2013), (Hespanha et al. 2007) of the network and the connectivity degradation (Mechraoui et al. 2011). So, it has been crucial to understand the impact of network failures on the system state and to consider them simultaneously with the control problems which characterize the co-design approach (Mechraoui et al. 2011). A diagnosis method is essential to identify and localize faults and to make the right decision to improve the system performance. Because of the stochastic behavior of wireless networks, probability theory is used. So, a Bayesian network BN is proposed to model the dependencies between failures and quantify their likelihood. Bayesian methods provide a formalism for reasoning under conditions of uncertainty (Pearl 2014). Many Bayesian models have been developed to perform diagnosis (Bottone et al. 2008), (Cheng et al. 2013), (Schumann et al. 2012). In this paper, the Bayesian model is inspired from the multi-layer model of Przytula \& Choi (2007). The proposed $\mathrm{BN}$ is used to identify the cause of QoC degradation of a networked mobile robot, whether it comes from the network as a system component susceptible to malfunctions, or the controller or the internal state of the robot. It is used also to make the decisions to avoid QoS and QoC degradation. Simon et al. (2013) and Mechraoui et al. (2011) propose, in the context of the co-design approach, the reconfigu- 
ration of the network to improve the QoC state or the adaptation of control in the case of QoS degradation. In the present work, a simulated networked mobile robot moving to a target by avoiding obstacles and controlled by a fixed station is considered. It is used to show the effectiveness of the proposed $\mathrm{BN}$ to diagnose and to make decisions to improve system performance. The paper is organized as follows. Section 2 is devoted to review basic theories of BNs. Section 3 describes the developed $\mathrm{BN}$ for the diagnosis of networked mobile robot. Diagnosis scenario is then presented to illustrate the relevance of BN. Finally, conclusions and perspectives are given in section 5 .

\section{BACKGROUND}

\subsection{Bayesian network $B N$}

BNs, also called beliefs networks, are probabilistic graphical model representing dependencies between events with their likelihood. They are the results of research work of Judea Pearl in the 80s (Pearl 2014). BNs have been used in many applications such as decisions making problem in autonomous vehicles, risk modeling and especially diagnosis which is the objective of this work. One of the advantage of this model is to gather and merge different types of data (Naïm et al. 2007). BNs are directed acyclic graphs in which the nodes represent variables (discrete or continuous) and the arcs represent direct causal influences between the connected variables. These influences, which represent the dependencies between the variables, are expressed by forward conditional probabilities. Finally, a BN is defined as follows:

- a directed acyclic graph $\mathrm{G}=(\mathrm{V}, \mathrm{E})$ where $\mathrm{V}$ is the set of nodes and $\mathrm{E}$ is the set of directed edges;

- a probability space $(\Omega, \mathrm{Z}, \mathrm{P})$;

- a set of random variables associated to the graph nodes such as

$$
P\left(V_{1}, V_{2}, \ldots, V_{n}\right)=\prod_{i=1}^{n} P\left(V_{i} \mid C\left(V_{i}\right)\right)
$$

where $C\left(V_{i}\right)$ is the set of $V_{i}$ parents.

An important concept in BNs is d-separation which helps to reduce probabilities calculations. If two variables are d-separated to a set of random variables $\mathrm{Y}$, then they are conditionally independent on $\mathrm{Y}$ in all probability distributions of the BN. Another characteristic of BNs is their ability to update beliefs via new evidence propagation in the network. The inference in BNs consists in updating the probabilities of unobserved variables after observations of a certain number of other variables values. Many algorithms are used to calculate the posterior probabilities such as the junction tree algorithm developed by Jensen. The exact inference in belief network is NP-hard.

\subsection{Distributed Bayesian Network}

Distributed BNs have been introduced in many works (Sayed and Lohse 2013), (Xiang and Lesser 2000), (Bloemeke and Valtorta 2002), (Langevin 2010), (Hwang and Cho 2006), (Hwang and Cho 2009) to shorten inference time, to reduce load on the calculations server and to infer information from different environments. In fact, the information coming from remote and smart devices should be preprocessed and inferred locally (Frank et al. 2014). The local inference results coming from different modules are then gathered for final inference. Three types of distributed $\mathrm{BN}$ have been studied. Multi Sectioned Bayesian Networks MSBN (Xiang and Lesser 2000) is a knowledge representation formalism that divides a large $\mathrm{BN}$ into sub-networks to enable multi-agent probabilistic reasoning. It is used to model a domain using a set of Bayesian subnet that are each assigned to a subset of the domain. The d-separation is used to section the global BN and all subnets are organized in a hyper tree structure. Besides, there is only one probability distribution for the set of all random variables of all local BNs. The constraints of MSBN limit the autonomy of the agents that must be tightly coupled. Regarding Agent Encapsulated Bayesian Networks (Bloemeke and Valtorta 2002), (Langevin 2010), each agent implements its internal knowledge as a local BN which has its own probability distribution. It uses its local $\mathrm{BN}$ for its own reasoning. These type of BNs modeling deals with problems where the information and the actors are distributed. The agents are organized in a publisher-subscriber hierarchy and communicate by passing messages through shared variables. The subscriber updates its beliefs based on its own observations and on the publisher beliefs because the publishing agent are more knowledgeable about the variables they produce, i.e. the subscribing agent replaces its current view of the shared variables with the publishers one. Another distributed Bayesian approach is developed by Hwang and Cho (2006, 2009). The BNs, in their approach, are modularized according to their separated sub-domains. The inference consists of two stages considering the co-causality of the modularized BN. Every Bayesian module infers in first given the available hard evidence. The first stage output of Bayesian modules that are connected as parents is introduced as soft evidence in the second stage by virtual linking.

In this work, Modular BN based on two-stage inference of Hwang and Cho is proposed to achieve the objective of diagnosis. A detailed description is introduced in section 3 .

\subsection{Bayesian network for diagnosis}

System diagnosis is the identification of failures in order to make decisions to return the system to its normal state. It consists in determining whether a com- 
ponent is failing or not i.e. localizing the origin of the failures from observations. A failure is defined as a deviation, outside an acceptable range, of an observed variable. The diagnosis task is divided into 3 subtasks (Patton and Chen 1999):

- failure detection which consists in observing system malfunction;

- failure localization to determine the failing component;

- failure identification which lies in the determination of the failure form and its amplitude.

BNs for diagnosis have been used in many fields. As probabilistic models, they evaluate the state of systems that have random dynamic and represent the dependencies between failures. They model the causal links that relate errors to symptoms based on human experts and engineering knowledge about the system. The inference algorithm is used to infer error causes from observed symptoms. Bottone et al. (2008) proposed a Bayesian model for satellite diagnosis. Their system consists of 4 types of component susceptible to failures. The model is composed of 2 main nodes types: a measurable node which corresponds to the state of a measurable parameter and a non measurable node defined as failure node which represents a component failure or a system failure. The failure node had 2 states: fail state for component failure and no fail state for operational component. The diagnosis procedure in this model determine the maximum likelihood of non measurable nodes given the state of measurable nodes and consequently the cause of system dysfunction. Cheng et al. (2013) developed a dynamic BN for the maintenance of Automatic Train Protection Control Unit ATPCU and for the diagnosis of the cause of train stop. In their model, a monitor node supervise the system and when it detects a failure, it pass the information to maintenance node. Consequently, the maintenance probability increases until a defined value that triggers the maintenance operation to upgrade the ATPCU state. A stop node gives the state of the train. If the train is stopped, the ATPCU is in degraded mode. A system condition node describes the conditions and the environment in which the system is operating. Another BN is used in NASA as Software Health Management for the diagnosis of software and hardware failures. Schumann et al. (2012) developed a modular, hierarchical and reusable Bayesian model which consists of discrete nodes. The command node $\mathrm{C}$ presents, as an observable node and an input to the $\mathrm{BN}$, a ground truth, a command, an action or a specific operating mode. The sensor node $\mathrm{S}$, which is also an observable node and an input to the $\mathrm{BN}$, gives measures about the software and hardware monitoring. A non observable node which is the Health node $\mathrm{H}$ is the result of diagnosis of the component state. The state of this node is thus
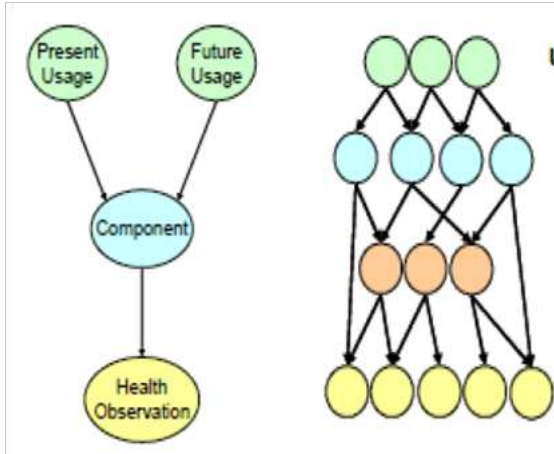

usage observations

components

sub systems

health

observations

Figure 1: Multi-layer Bayesian network for diagnosis

the output of the model. The developed model was applied to the diagnosis of an autonomous robot and an aircraft control system. Another Bayesian model for diagnosis, from which the proposed Bayesian network is inspired, is the multilayer model of Przytula and Choi (2007) as shown in figure 1. The first layer is the usage layer where observable nodes present the actual system usage or its operating mode. The layer component gathers nodes describing components or their failure modes whose states are the result of diagnosis. The subsystems layer can be added to represent the state of a subsystem corresponding to a subset of components. Finally, the health observations layer contains observable nodes to monitor the components states. Such a structure reduces the number of needed parameters to define the BN. The objective is to diagnose the components state from the health observations and system usage. The previous Bayesian models, mainly the last one, have been studied in this work to develop a consistent $\mathrm{BN}$ for diagnosis of networked mobile robot which is detailed in the next section.

\section{CONSTRUCTING THE BN MODEL}

The global system consists of a robot equipped with actuators and sensors and communicating to a remote station where the controller is implemented. The mobile robot has limitations such as insufficient memory capacity, lower CPU power and limited battery lifetime. The defaults that have an impact on the system QoC have been studied. In this work, the QoC measures the capacity of the controller to bring the robot to its steady position via wireless network. The QoC is affected by the state of the network, as a system component, and more specifically, its QoS. Besides, the robot state has an impact on the QoC because in the case of actuators degradation, the robot won't be able to execute the station orders. The global system is divided into 3 sub-systems which are the network sub-system, the operative sub-system (robot actuators and sensors) and the control sub-system. For each sub-system, a BN is modeled to represent the dependencies between failures of each sub-domain. Consequently, a modular $\mathrm{BN}$ for diagnosis is constructed in order to identify the QoC degradation cause whether it is network failure, or a robot failure or a controller 


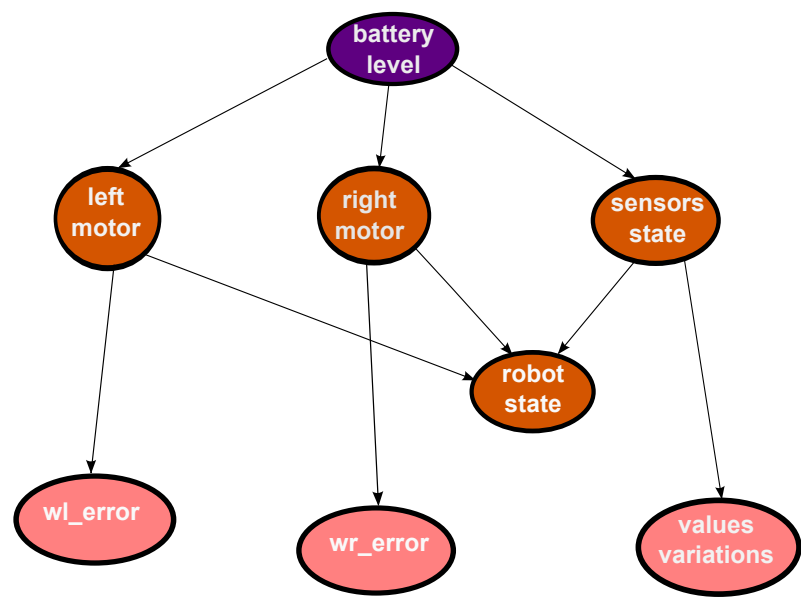

Figure 2: Operative Bayesian module

failure. Each BN module is structured in a multilayer model like the Przytula and Choi (2007) model to provide more accurate and broad coverage context specially the components level, the intermediate one, which represents relationships between component failures. The first layer represents the usage of the system and the conditions in which it is running. There is also another intermediate layer which is the subsystem layer representing the state of each subsystem of the global one. Finally, observations layer is used to monitor the state of components or components failures. The operative Bayesian module is implemented on the robot while the other two modules are on the station side.

\subsection{Operative Module}

The robot is equipped with sensors and motors (actuators) which represent the operative subsystem of the WNCS. For this subsystem, a subsystem node is defined as robot state to indicate the state of this part of the system as shown in figure 2. The components (components node) of this subsystem are the left and right motor and the obstacles sensors of the robot. For each component node, an observation node measures and monitors its health state. The sensors are considered failing if they are not able to detect the presence of obstacles or the transmitted values are constant or incorrect. For the actuators, a motor failure is monitored by observing the reference error of the angular velocity. This velocity error must decrease over time and does not exceed a threshold, otherwise, the motor (left or right) is failing. A usage node battery level is used to describe the robot battery usage, it represents the conditions in which the robot components are operating.

\subsection{Network module}

The network is considered as a WNCS component which can be failing like the other system components. When the network cannot ensure the required QoS, it is considered failing. The network state is represented by the node QoS which is a subsystem node as shown in figure 3. The network parameters that have an impact on the QoS are packets loss rate, delay, jitter and throughput. Failures which are associated to this observable parameters are defined to describe the degradation of the QoS state. There are 3 types of failure mode in the network when some communicating device are mobile: channel failure, transmission failure and connectivity failure. For the communication channel failure which limits the network availability, a component node channel state is defined because when the channel is saturated, there is no guarantee that the message will be transmitted. To monitor the channel state, an observation node throughput measures the rate of transmitted packets in the network. Regarding the connectivity failure, when the mobile robot moves away from the station coverage area, it loses its connectivity to the station so the messages are dropped. To observe the state of connectivity node, the received signal strength indicator RSSI is used to measure the quality of the received signal. The nodes deadline respect and data loss are devoted to transmission failure description. The jitter and delay measurement monitors the reception on time of the transmitted packets and the packet loss observes the rate of dropped packet in the network. A set of nodes in the usage layer define the use conditions of the network. Number of communicating nodes define the number of mobile communicating entities in the network. If this number increases, the collision probability increases so the throughput decreases, the delay and packet loss rate increase. The node network load represents the network limited resources offered to the application. If the network is overloaded, its performances degrades, the delay and packet loss increase and the channel is saturated. The closed loop sampling period has also an impact on the network state because if its value is under a certain threshold, the network will be overloaded and packets will consequently be dropped (Simon et al. 2013). The connectivity state depends on the environment in which the robot is moving, where there are obstacles (obstacle node)and a risk of signal fading or absorption. It depends also on the distance between the station and the robot to define the coverage area where the connection persists.

\subsection{Control module}

The QoC node, which represents the control subsystem, indicates the performance and the state of the control application, i.e. it indicates whether the robot is controlled via wireless network or not. The QoC is influenced by the robot state (state of operative subsystem), the QoS state (state of the network subsystem) and the state of the controller as shown in figure 4. If the robot actuators don't receive on time the control data or they are not able to execute the control laws, the robot cannot be well controlled therefore the QoC state degrades. Observations node are defined to 


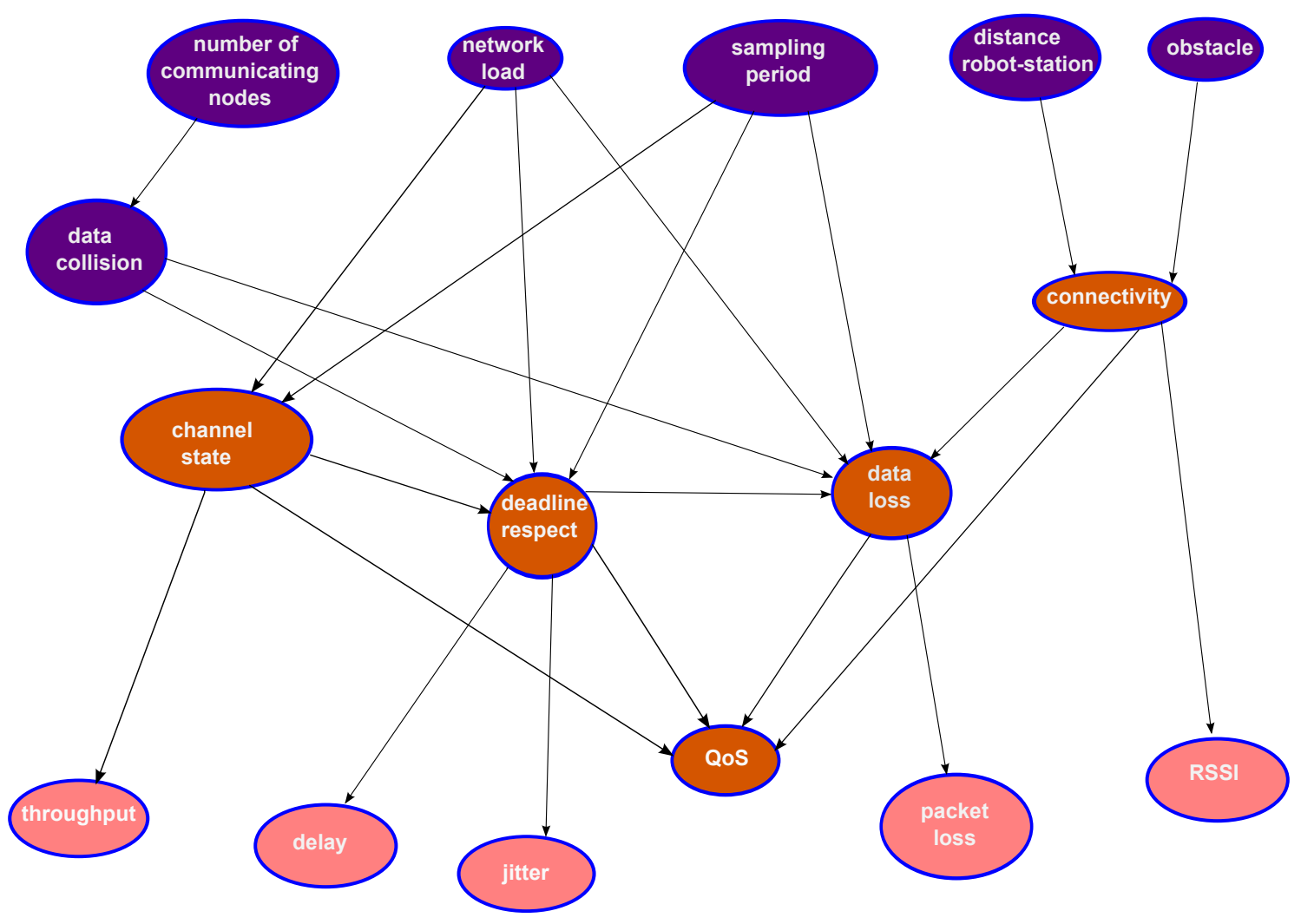

Figure 3: Network Bayesian Module

monitor the QoC state. The stability node controls the system stability along the trajectory using Lyapunov theory. The position error node monitors the distance between the target and the robot (navigation mission) which must decrease over time. The distance robotobstacle node monitors the distance between the robot and an obstacle (if it exists in the robot trajectory) because the robot must reach the target position while avoiding obstacles. The controller node, which is a component node, has an impact on the QoC state. If the controller has limited calculations capacity or its CPU is overloaded, the QoC degrades. Two usage nodes are defined for the controller to precise its type and its CPU load. The embedded controller (implemented on the robot) has degraded performance while the remote controller (station) is efficient.

\subsection{Diagnosis reasoning}

The Bayesian modules are used to perform networked mobile robot diagnosis in order to maintain it in a good state and lead it to its target. In this model, the control subnet shares with the network and operative subnets the QoS node and the robot state node respectively. In the first inference stage, hard evidence are applied in 3 modules. Secondly, the result of the inference in the control subnet is transferred to the other two subnets as soft evidence on the Qos node and robot state node. In the next section, diagnosis scenarios are described to prove the relevance of these method using a simulated networked robot controlled by a remote station.

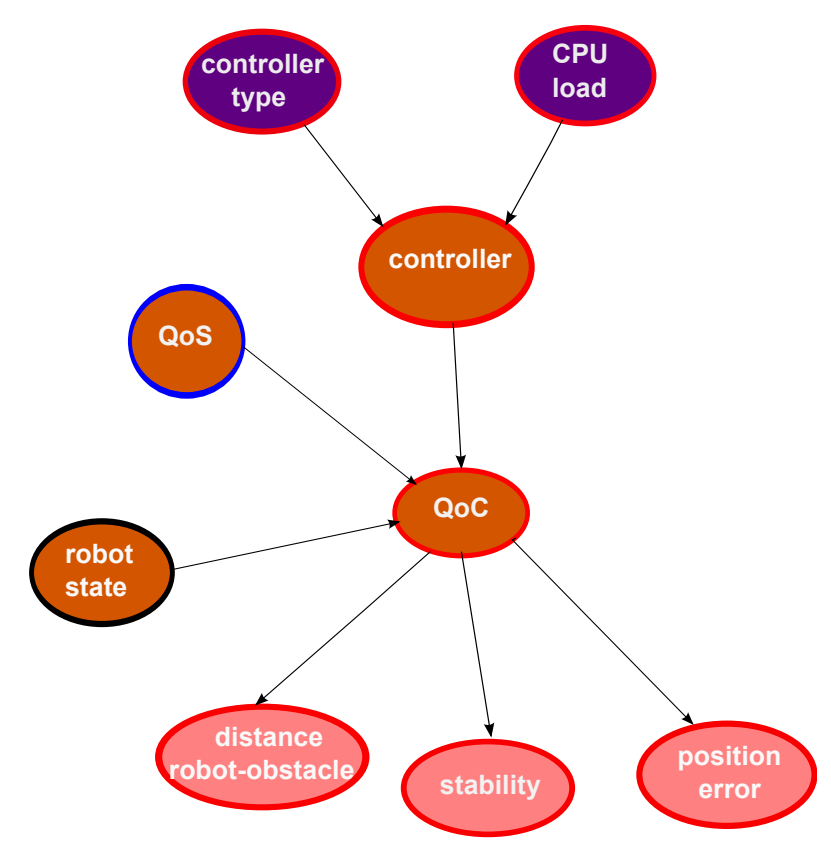

Figure 4: Control Bayesian Module

\section{DIAGNOSIS SCENARIO}

A diagnosis scenario is presented below to verify the efficiency of the developed approach to decisions making. Based on the QoC state in the one hand and on the QoS state on the other hand, the remote station orders the robot to switch to its embedded controller if it is necessary. Algorithm 1 is used as a strategy to control the robot in distance using the Bayesian diagnosis results after entering observations on the BN. Truetime toolbox (2.0 version) (Cervin et al. 2010) is used to co-simulate (network and control cosimulation) the networked robot controlled by a re- 


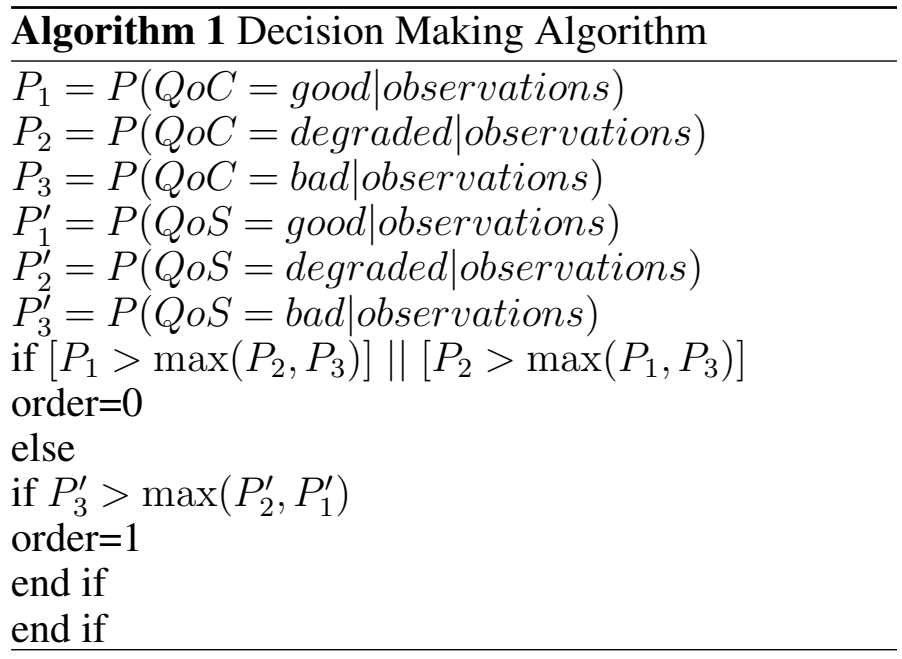

mote station whose position is $(1,1)$ and BNT toolbox (Murphy et al. 2001) is used to implement the BN. The robot starts from its initial position $(\mathrm{x} 0, \mathrm{y} 0)=(0,0)$ to the target one $(x d, y d)=(15,1)$ as shown in the figure 5 where it moves in the station coverage zone and in good network conditions. The robot reaches its target after 60s when the linear velocity becomes zero. A network fault occurs, the network delay is greater than the sampling period of the control loop which is equal to $0.4 \mathrm{~s}$, the application performance is degraded and the QoC state becomes bad because of the degradation of the QoS. No actions are taken in the case of figure 6 which shows that the robot cannot be sta-
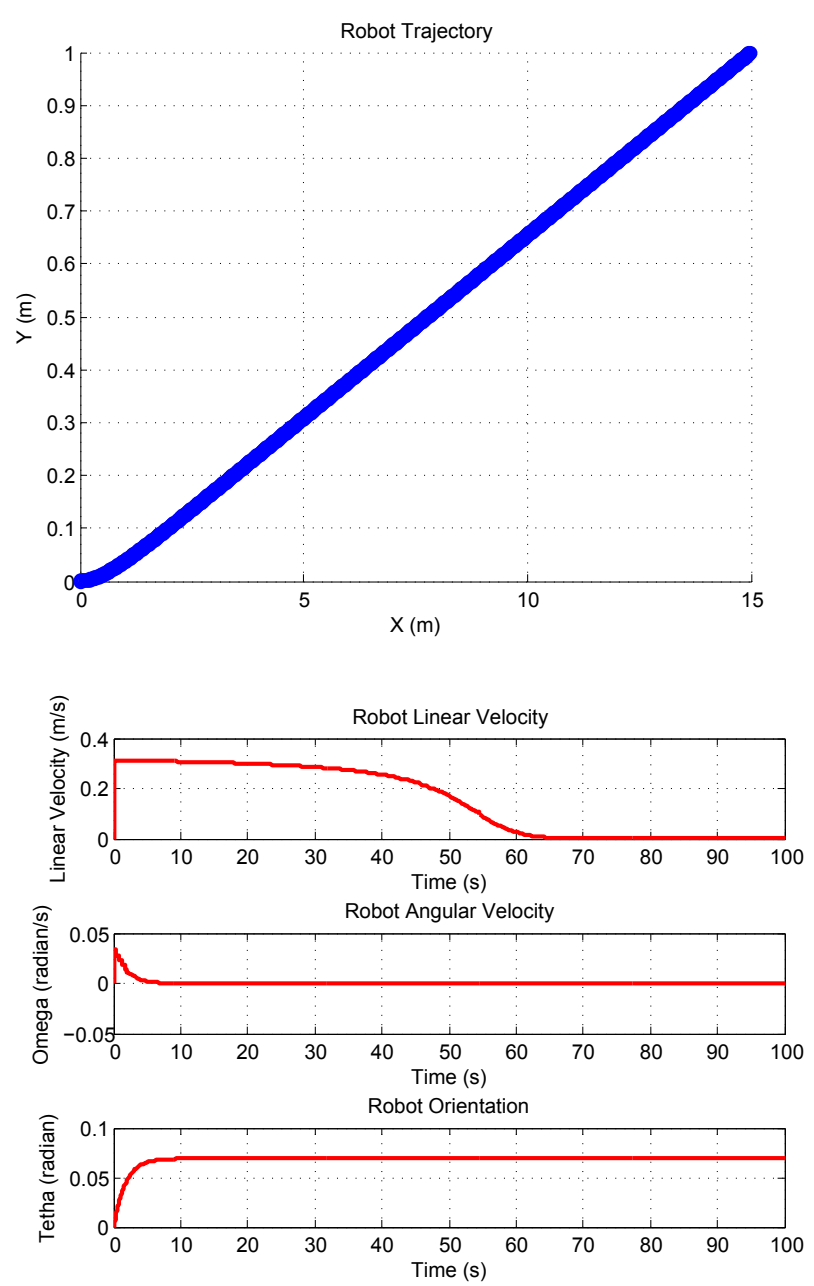

Figure 5: Robot navigation in good network conditions
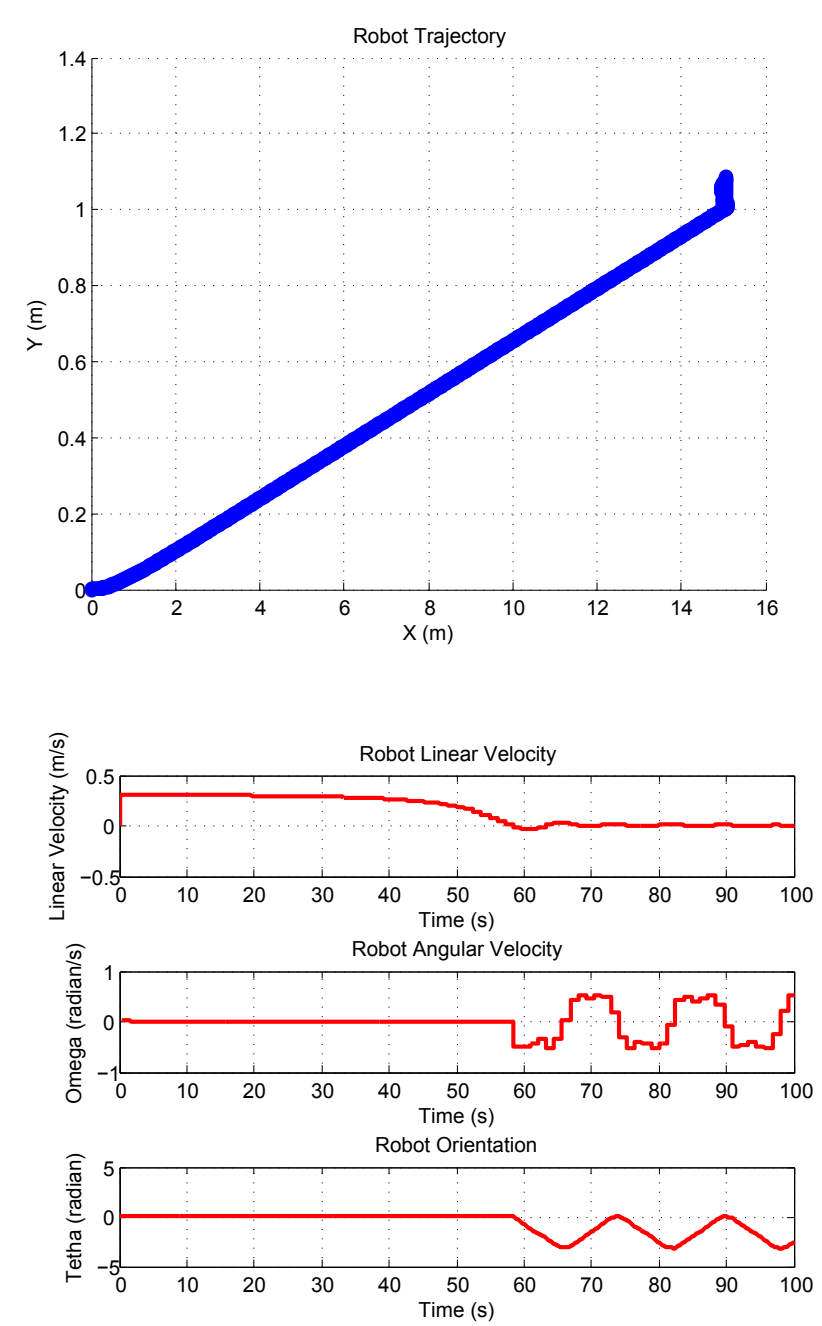

Figure 6: Robot navigation in degraded network conditions

ble on its steady position $(15,1)$. Adopting a co-design approach action, the algorithm 4 is executed based on the $\mathrm{BN}$ results to switch to embedded controller in case of QoC degradation caused by the QoS bad state. The robot takes an order from the station and reaches the target position based on its embedded controller as shown in figure 7 .

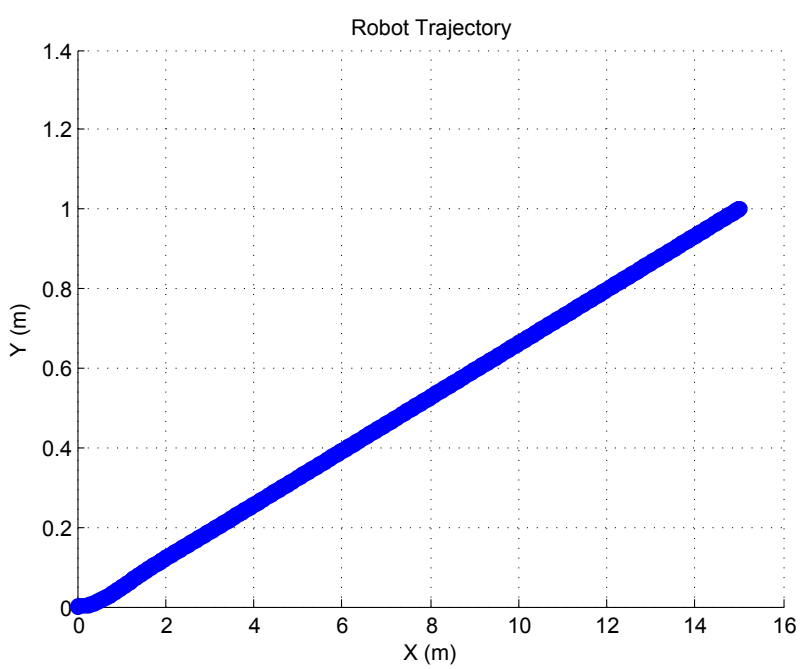

Figure 7: Robot navigation with switching to embedded controller 
In this article, a diagnosis method for wireless networked mobile robot based on modular $\mathrm{BN}$ is proposed in order to maintain the controlled system operational and lead it to its target. This approach takes into consideration both QoS and QoC states to solve a co-design problem. The developed method is then tested on a simulated networked mobile robot. Probabilities given by the $\mathrm{BN}$, describing the actual situation, generate an order to maintain the communication between the robot and the station or to make controllers switching decision. This strategy gives first satisfying result in term of application performance because the robot reaches its target after switching to embedded controller. In this work, decisions have been made based on QoS and QoC states. In further work, the intermediate layer of each $\mathrm{BN}$ will be used to make more efficient decisions based on each type of network failure, on the controller state or on each robot component state. The developed Bayesian method will be implemented on a real mobile robot. The prognostic aspect will be also integrated in this work to avoid QoC and QoS degradation before its occurrence.

\section{REFERENCES}

Berbra, C., S. Gentil, S. Lesecq, \& J.-M. Thiriet (2008). Codesign of a safe network control quadrotor. In Proceedings of 17 th IFAC World Congress, Volume 8.

Bloemeke, M. \& M. Valtorta (2002). Agent-encapsulated bayesian networks and the rumor problem. Technical report, Technical Reports, TR 2002-006, Depart of Computer Science, University of South Carolina.

Bottone, S., D. Lee, M. O’Sullivan, \& M. Spivack (2008). Failure prediction and diagnosis for satellite monitoring systems using bayesian networks. In Military Communications Conference, 2008. MILCOM 2008. IEEE, pp. 1-7. IEEE.

Cervin, A., D. Henriksson, \& M. Ohlin (2010). Truetime 2.0 betareference manual. Department of Automatic Control, Lund University (June 2010).

Cheng, Y., T. Xu, \& L. Yang (2013). Bayesian network based fault diagnosis and maintenance for high-speed train control systems. In Quality, Reliability, Risk, Maintenance, and Safety Engineering (QR2MSE), 2013 International Conference on, pp. 1753-1757. IEEE.

Frank, K., M. Röckl, T. Pfeifer, \& P. Robertson (2014). Segmenting bayesian networks for intelligent information dissemination in collaborative, context-aware environments with bayeslets. Pervasive and Mobile Computing 12, 214-231.

Ghostine, R., J.-M. Thiriet, \& J.-F. Aubry (2011). Variable delays and message losses: influence on the reliability of a control loop. Reliability Engineering \& System Safety 96(1), 160-171.

Hespanha, J. P., P. Naghshtabrizi, \& Y. Xu (2007). A survey of recent results in networked control systems. Proceedings IEEE 95(1), 138.

Hwang, K.-S. \& S.-B. Cho (2006). Modular bayesian networks for inferring landmarks on mobile daily life. In AI 2006: Advances in Artificial Intelligence, pp. 929-933. Springer.

Hwang, K.-S. \& S.-B. Cho (2009). Landmark detection from mobile life log using a modular bayesian network model. Expert Systems with Applications 36(10), 12065-12076.

Langevin, S. (2010). Knowledge representation, communica- tion, and update in probability-based multiagent systems. In Proceedings of the 9th International Conference on Autonomous Agents and Multiagent Systems: volume 1-Volume 1, pp. 1663-1664. International Foundation for Autonomous Agents and Multiagent Systems.

Lian, F.-L., J. Moyne, \& D. Tilbury (2002). Network design consideration for distributed control systems. Control Systems Technology, IEEE Transactions on 10(2), 297-307.

Mechraoui, A., F. Djohor, J.-M. Thiriet, \& S. Gentil (2011). A co-design distributed bayesian approach for decision and scheduling of wncs. In 18th IFAC World Congress (IFAC WC 2011), pp. 14970-14975.

Mechraoui, A., Z. H. Khan, \& J.-M. Thiriet (2009). Effect of packet loss on the quality of control of a networked mobile robot. In 30th IFAC Workshop on Real-Time Programming and 4th InternationalWorkshop on Real-Time Software, pp. 97-101.

Moyne, J. R. \& D. M. Tilbury (2007). The emergence of industrial control networks for manufacturing control, diagnostics, and safety data. Proceedings of the IEEE 95(1), 29-47.

Murphy, K. et al. (2001). The bayes net toolbox for matlab. Computing science and statistics 33(2), 1024-1034.

Nagatani, K., S. Kiribayashi, Y. Okada, K. Otake, K. Yoshida, S. Tadokoro, T. Nishimura, T. Yoshida, E. Koyanagi, M. Fukushima, et al. (2013). Emergency response to the nuclear accident at the fukushima daiichi nuclear power plants using mobile rescue robots. Journal of Field Robotics 30(1), 44-63.

Naïm, P., P. Wuillemin, P. Leray, O. Pourret, \& A. Becker (2007). Les réseaux bayésiens. (3 éme édition ed.). Paris: Eyrolles.

Patton, R. J. \& J. Chen (1999). Robust model-based fault diagnosis for dynamic systems.

Pearl, J. (2014). Probabilistic reasoning in intelligent systems: networks of plausible inference. Morgan Kaufmann.

Przytula, K. W. \& A. Choi (2007). Reasoning framework for diagnosis and prognosis. In Aerospace Conference, 2007 IEEE, pp. 1-10. IEEE.

Sayed, M. S. \& N. Lohse (2013). Distributed bayesian diagnosis for modular assembly systemsa case study. Journal of Manufacturing Systems 32(3), 480-488.

Schumann, J., T. Mbaya, \& O. J. Mengshoel (2012). Software and system health management for autonomous robotics missions. Proc. of $i$-SAIRAS 2012.

Simon, D., Y.-Q. Song, \& C. Aubrun (2013). Co-design approaches to dependable networked control systems. John Wiley \& Sons.

Tipsuwan, Y. \& M.-Y. Chow (2003). Control methodologies in networked control systems. Control engineering practice 11(10), 1099-1111.

Xiang, Y. \& V. Lesser (2000). Justifying multiply sectioned bayesian networks. In IEEE (Ed.), Proceedings Fourth International Conference, pp. 349-356. IEEE.

Zhang, L., H. Gao, \& O. Kaynak (2013). Network-induced constraints in networked control systemsa survey. Industrial Informatics, IEEE Transactions on 9(1), 403-416. 